\title{
Combination of sorafenib, vorinostat and bortezomib for the treatment of poor-risk AML: report of two consecutive clinical trials
}

Hamid Sayar ${ }^{1}$, Larry D. Cripe ${ }^{1}$, Antoine N. Saliba², Mohammad Abu Zaid1, Heiko Konig1, H. Scott Boswell 1,3

1. Indiana University Melvin and Bren Simon Cancer Center, Indiana University School of Medicine, Department of Medicine, Division of Hematology/Oncology, Indianapolis, IN, USA 2. Department of medicine, Indiana University School of Medicine, Indianapolis, IN, USA

3. Veterans Affairs Medical Center, Indianapolis, IN, USA

Corresponding author:

Hamid Sayar, MD, MS

Indiana Cancer Pavilion

535 Barnhill Drive, RT 473

Indianapolis, IN 46202

USA

Email: ssayar@iu.edu

Keywords: Acute myeloid leukemia, AML, sorafenib, vorinostat, bortezomib

This is the author's manuscript of the article published in final edited form as:

Sayar, H., Cripe, L. D., Saliba, A. N., Abu Zaid, M., Konig, H., \& Boswell, H. S. (2018). Combination of sorafenib, vorinostat and bortezomib for the treatment of poor-risk AML: report of two consecutive clinical trials. Leukemia Research. https://doi.org/10.1016/j.leukres.2018.12.011 


\section{Introduction}

Older age, unfavorable cytogenetics, and FLT3-ITD mutation are adverse prognostic indicators in acute myeloid leukemia (AML) placing the disease under poor-risk category [1-5]. Complex biologic factors in poor-risk AML confer resistance to chemotherapy [6-9]. Incorporation of novel non-chemotherapeutic targeted agents into the treatment of poorrisk AML is therefore on demand. Subject of such approaches may include epigenetic pathways that affect tumor suppressor genes. Apoptosis in leukemic blasts can be promoted through upregulation of tumor suppressors DAPK1, RUNX3, and p16INK4A/CDKN2A $[10,11]$. We previously identified an oncogenic pathway in AML repressing promoter of DAPK1 gene through an interaction of p52NF- $\kappa B$ and histone deacetylases [12]. Furthermore, leukemia stem cell promotion occurs in epigenetically repressed FLT3-ITD positive (+ve) or complex cytogenetics disease [13]. Indeed, a cause for suboptimal response to single-agent FLT3 inhibitors may be an epigenetic collaboration in the pathogenesis of FLT3-ITD-mediated AML [14,15].

We hypothesized that epigenetic de-repression of specific tumor suppressor genes in addition to inhibition of kinase-dependent proliferation systems can promote clinical antileukemic effect in AML. We therefore designed sequential clinical trials to explore the safety and efficacy of epigenetic modification along with targeting selected cell proliferation pathways in poor-risk AML. The first trial combined FLT3/RAF inhibitor sorafenib and histone deacetylase inhibitor vorinostat in a phase 1 setting. The second trial tested addition of bortezomib to the previous combination for further tumor suppressor derepression through inhibition of p52NF- $\mathrm{kB}$. Our findings on the molecular attributes of these studies have been reported separately [16]. This report describes clinical aspects of the two consecutive trials.

\section{Patient selection}

Both studies were approved by Indiana University School of Medicine Institutional Review Board. Sor/vor study included adult patients with a diagnosis of relapsed and/or refractory AML or older than 70 with previously untreated disease. Any cytogenetic or molecular profile was acceptable. The sor/vor/bor study enrolled only relapsed and/or refractory AML with FLT3-ITD+ve profile and/or poor-risk cytogenetics including -5, -7, or complex 
karyotype. Such selection was due to observation of response in patients with poor-risk cytogenetics or FLT3-ITD+ve disease in sor/vor trial.

\section{Trial Design and Treatment}

The sor/vor trial followed a 3+3 dose-escalation schedule in a phase I setting. Sorafenib was given orally at a dose of $400 \mathrm{mg}$ twice daily, and oral vorinostat was given with dose escalation in successive cohorts. Each cycle of 21 days consisted of treatment for 14 days followed by 7 days of break. An interrupted schedule was chosen to avoid excessive myelosuppression. Patients who achieved at least $50 \%$ reduction of bone marrow blasts with the first cycle, were eligible to receive the second cycle. At least $10 \%$ reduction of bone marrow blasts, but not a complete remission with the second cycle, would make patients eligible for the third cycle. A maximum of 3 treatment cycles was planned. No further treatment would be given beyond a complete remission (CR).

Sor/vor/bor was a phase I/II study with a 3+3 dose-escalation schedule in the phase I part. Vorinostat was given orally at $200 \mathrm{mg}$ twice daily, initially in an interrupted fashion only on days 1-4 and 8-12, and later continuously. Oral sorafenib and intravenous bortezomib were given with dose escalation in successive cohorts. Each cycle consisted of 14 days of treatment followed by 7 days of break, 21 days total. Patients with continued response would continue on study. Up to 2 more cycles could be given beyond a CR. The phase II part of the study followed the same schedule of phase I, given at safe dose determined by the phase I part.

Response was assessed by the guidelines reported by Cheson [17], and dose-limiting toxicity (DLT) was defined as any grade 3 or 4 non-hematologic toxicity.

\section{Results}

\section{First trial (sor/vor study)}

Toxicity and optimal dose. Patient characteristics are summarized in table 1 . A total of 15 patients were enrolled, and 13 completed at least one cycle of therapy. Median age was 61 years (37-74), with 8 males and 5 females. Two patients were in their $3^{\text {rd }}$ relapse, one had 
relapsed after allo-transplant, one older patient had previously untreated disease, and rest of patients were refractory to chemotherapy. One patient had grade 1 hand-foot syndrome, and another grade 2 nausea. One/six patient in the third (last) cohort developed grade 4 diarrhea in the setting of neutropenic fever. The safe dose was determined as sorafenib 400 $\mathrm{mg}$ and vorinostat $200 \mathrm{mg}$ both twice daily for 14 days, followed by 7 days of rest. To further assess the safety and response, three more patients were enrolled into the study at the established optimal dose.

Response. With the first cycle of treatment, seven/sixteen (44\%) patients demonstrated a partial remission (PR) and 1/16 (6\%) achieved a complete remission (CR) and remained disease-free for 5 months. Four partial responders received 1-2 more cycles with initial response but experienced disease progression. Three partial responders to the first cycle did not receive a second cycle, one per personal preference and two due to increasing circulating blasts. The patient with CR carried a complex cytogenetics including monosomies 5 and 7.

\section{Second trial (sor/vor/bor study)}

Toxicity and optimal dose. Seventeen patients were enrolled in the phase I part (table 2). Fifteen patients completed at least one cycle of treatment. All patients had relapsed and/or refractory disease, and had received at least two lines of chemotherapy. Median age was 51 years (24-73), with 10 males and 7 females. Most common toxicities were gastrointestinal disturbances (64.2\%) including diarrhea, nausea, or vomiting reaching grade 2 in $14.2 \%$ of patients with no grade 3 or 4 . Others included fatigue (31.3\%), rash (21.4\%), and neutropenic fever (7.1\%); none greater than grade 2 . The safe dose was sorafenib $400 \mathrm{mg}$ and vorinostat $200 \mathrm{mg}$ twice daily for 14 days, and bortezomib $1.3 \mathrm{mg} / \mathrm{m}^{2} \mathrm{IV}$ on days $1,4,8$, 11 , every 21 days.

Response. In the phase I study 5 patients achieved CR with incomplete platelet recovery (CRi). Responses occurred across different cohorts. A total of 20 patients were enrolled into the phase II study. Majority of patients were heavily pretreated including stem cell transplantation in 5. Six patients were deemed unevaluable for response. Of 14 evaluable patients, 7 (50\%) carried FLT3-ITD mutation and 7 (50\%) had either complex cytogenetics or monosomy 7. One patient (7\%) achieved CR, 1 (7\%) CRi, and 2 patients (14\%) 
experienced a PR. Overall responses with all 37 patients in both phases, including unevaluable patients, were 1 CR (3\%), 6 CRi (16\%), and 2 PR (5\%). All responders in both phases of the study carried FLT3-ITD mutation. Responders all experienced relapse within 2 cycles of consolidation, and none was able to undergo transplant due to variety of reasons.

\section{Discussion}

The two consecutive studies were based on a hypothesis that epigenetic de-repression of tumor suppressor genes combined with inhibition of kinase-dependent proliferation systems can introduce a collaborative anti-leukemic effect. The first study demonstrated safety of combined kinase inhibitor sorafenib and epigenetic modulator vorinostat. In this study a clinical response, including 1 CR and 6 PR were observed in poor-risk AML. Analysis of bone marrow blasts in this study demonstrated up-regulation of tumor suppressors DAPK1, RUNX3, and CDKN2A along with reduction in FLT3-induced Id1 [16].

The second trial established safety and assessed efficacy of sorafenib, vorinostat, and bortezomib combination. Bortezomib was added to further enhance de-repression of tumor suppressors through inhibition of p52NF- $\mathrm{kB}$. In this study 1 CR (3), 6 CRi (16\%), and 2 PR (5\%) were observed. In this study also an upregulation of tumor suppressors of interest was noted in responders [16]. Maximum response in both studies occurred within the first 2 cycles of treatment. The depth of response, as is evidenced by number of CR and CRi, was greater in the second study, reflecting potential role of proteasome inhibition in this combination. Responses in both studies were distributed among different dose levels, suggesting sensitivity of leukemic cells to the combination regardless of clinical dosing.

Single-agent sorafenib has shown efficacy in AML when administered continuously, with median time to best response of 27 to 46 days $[18,19]$. Majority of responders in both of our studies achieved best response within the first 14 days of therapy. Indeed, intracellular molecular changes predictive of response were observed in the AML blasts obtained from patient marrows as early as 3-4 days following initiation of treatment [16]. The expedited response observed in our studies is logically attributable to addition of vorinostat and possibly bortezomib. 
Patients were not kept on treatment extensively due to limitations in drug availability, but all relapses in sor/vor/bor occurred during consolidations, indicating long-term treatment would not be beneficial. The short duration of response to sorafenib in FLT3-ITD+ve disease is attributed to emergence of D835 tyrosine kinase domain mutation [18]. Additional epigenetic modulation in our studies did not improve such response duration.

The two studies demonstrated safety of combination of sorafenib, vorinostat, and bortezomib at full approved doses in AML. These studies also showed potential for response to novel targeted-only therapies in poor-risk AML. These studies offer a platform towards further development of such rational combinations for the treatment of AML.

\section{Acknowledgments}

Authors would like to thank all patients who participated in these studies.

\section{Funding sources}

Studies were funded in part by a research grant from Bayer/Onyx, the Wendy Will Case Cancer fund, stipend in memory of Dr. Gary D. Tollefson, and Department of Veterans Affairs Merit Review Award.

\section{Conflict of interest}

Hamid Sayar received research funding from Bayer/ Onyx. Other authors declare no conflict of interests. 


\section{References}

[1]. Alibhai SM, Leach M, Minden MD, et al. Outcomes and quality of care in acute myeloid leukemia over 40 years. Cancer. 2009;115(13):2903-2911.

[2]. Menzin J, Lang K, Earle CC, et al. The outcomes and costs of acute myeloid leukemia among the elderly. Arch Intern Med. 2002;162(14):1597-1603.

[3]. Almeida AM, Ramos F. Acute myeloid leukemia in the older adults. Leuk Res Rep. 2016 Jun 16;6:1-7.

[4]. Orozco JJ, Appelbaum FR. Unfavorable, Complex, and Monosomal Karyotypes: The Most Challenging Forms of Acute Myeloid Leukemia. Oncology (Williston Park). 2012 Aug;26(8):706-12.

[5]. Kottaridis PD, Gale RE, Frew ME, et al. The presence of a FLT3 internal tandem duplication in patients with acute myeloid leukemia (AML) adds important prognostic information to cytogenetic risk group and response to the first cycle of chemotherapy: analysis of 854 patients from the United Kingdom Medical Research Council AML 10 and 12 trials. Blood. 2001 Sep 15;98(6):1752-9.

[6]. Leith CP, Kopecky KJ, Godwin J, et al. Acute myeloid leukemia in the elderly: assessment of multidrug resistance (MDR1) and cytogenetics distinguishes biologic subgroups with remarkably distinct responses to standard chemotherapy. A Southwest Oncology Group study. Blood. 1997 May 1;89(9):3323-9.

[7]. Willman CL. The prognostic significance of the expression and function of multidrug resistance transporter proteins in acute myeloid leukemia: studies of the Southwest Oncology Group Leukemia Research Program. Semin Hematol. 1997 Oct;34(4 Suppl 5):2533.

[8]. van den Heuvel-Eibrink MM, Wiemer EA, de Boevere MJ, et al. MDR1 expression in poorrisk acute myeloid leukemia with partial or complete monosomy 7. Leukemia. 2001 Mar;15(3):398-405.

[9]. Patel C, Stenke L, Varma S, et al. Multidrug resistance in relapsed acute myeloid leukemia: evidence of biological heterogeneity. Cancer. 2013 Aug 15;119(16):3076-83. [10]. Karlic H, Herrmann H, Varga F, et al. The role of epigenetics in the regulation of apoptosis in myelodysplastic syndromes and acute myeloid leukemia. Crit Rev Oncol Hematol. 2014 Apr;90(1):1-16.

[11]. Estécio MR, Maddipoti S, Bueso-Ramos C, et al. RUNX3 promoter hypermethylation is frequent in leukaemia cell lines and associated with acute myeloid leukaemia inv(16) 
subtype. Br J Haematol. 2015 May;169(3):344-51.

[12]. Shanmugam R, Gade P, Wilson-Weekes A, et al. A non-canonical Flt3ITD/NF-кB signaling pathway represses DAPK1 in acute myeloid leukemia (AML). Clin Cancer Res. 2012 Jan 15; 18(2): 360-369.

[13]. de Jonge HJ, de Bont ES, Valk PJ, et al. AML at older age: age-related gene expression profiles reveal a paradoxical down-regulation of p16INK4A mRNA with prognostic significance. Blood. 2009;114(14):2869-77.

[14]. Levis M, Ravandi F, Wang ES, et al. Results from a randomized trial of salvage chemotherapy followed by lestaurtinib for patients with FLT3 mutant AML in first relapse. Blood. 2011;117(12):3294-301.

[15]. Shih AH, Jiang Y, Meydan C, et al. Mutational cooperativity linked to combinatorial epigenetic gain of function in acute myeloid leukemia. Cancer Cell. 2015

[16]. Sayar H, Liu Y, Gao R, Zaid MA, et al. Consecutive epigenetically-active agent combinations act in ID1-RUNX3-TET2 and HOXA pathways for Flt3ITD+ve AML. Oncotarget. 2017 Dec 25;9(5):5703-5715.

[17]. Cheson BD, Bennett JM, Kopecky KJ, et al. Revised recommendations of the International Working Group for Diagnosis, Standardization of Response Criteria, Treatment Outcomes, and Reporting Standards for Therapeutic Trials in Acute Myeloid Leukemia. J Clin Oncol 2003;21(24):4642-9.

[18]. Man CH, Fung TK, Ho C et al. Sorafenib treatment of FLT3-ITD(+) acute myeloid leukemia: favorable initial outcome and mechanisms of subsequent nonresponsiveness associated with the emergence of a D835 mutation. Blood. 2012 May 31;119(22):5133-43. [19]. De Freitas T, Marktel S, Piemontese S, et al. High rate of hematological responses to sorafenib in FLT3-ITD acute myeloid leukemia relapsed after allogeneic hematopoietic stem cell transplantation. Eur J Haematol. 2016 Jun;96(6):629-36. 
Table 1. Sor/vor study patient characteristics

\begin{tabular}{|c|c|c|c|c|c|c|}
\hline Cohort & Patient & Age/Sex & AML status & Cytogenetics & FLT3-ITD & Response \\
\hline \multirow{3}{*}{1} & 1 & $71 / \mathrm{M}$ & $3^{\text {rd }}$ rel & Normal & - & No \\
\hline & 2 & $56 / \mathrm{M}$ & Rel, post transplant & Normal & - & No \\
\hline & 3 & $65 / \mathrm{M}$ & Primary ref & -7 & - & PR \\
\hline \multirow{3}{*}{2} & 4 & $72 / \mathrm{M}$ & $3^{\text {rd }}$ rel & $\mathrm{t}(1 ; 6)$ & - & No \\
\hline & 5 & $72 / \mathrm{M}$ & Rel/ref & $\mathrm{t}(18 ; 21)$ & + & $\mathrm{PR}$ \\
\hline & 6 & $37 / \mathrm{F}$ & Rel/ref & Normal & - & No \\
\hline \multirow{10}{*}{3} & 7 & $58 / \mathrm{M}$ & Rel/ref & Normal & + & $\mathrm{PR}$ \\
\hline & 8 & $74 / \mathrm{M}$ & Untreated & $-5,-7$ & - & $\mathrm{CR}$ \\
\hline & 9 & $46 / \mathrm{F}$ & Rel/ref & Complex & - & No \\
\hline & 10 & $74 / \mathrm{M}$ & Primary ref & Complex & - & $\mathrm{PR}$ \\
\hline & 11 & $60 / \mathrm{F}$ & Primary ref & Normal & - & No \\
\hline & 12 & $70 / \mathrm{F}$ & Untreated & Normal & - & $\mathrm{PR}$ \\
\hline & 13 & $42 / \mathrm{F}$ & Primary ref & Normal & + & $\mathrm{PR}$ \\
\hline & 14 & $53 / \mathrm{M}$ & $2^{\text {nd }}$ rel & Trisomy 8 & - & No \\
\hline & 15 & $67 / \mathrm{M}$ & Rel/ref & Normal & - & No \\
\hline & 16 & $70 / \mathrm{F}$ & Rel/ref & Normal & - & PR \\
\hline
\end{tabular}

Rel: Relapsed; Ref: Refractory; PR: Partial remission; CR: Complete remission

Dosing: Sorafenib $400 \mathrm{mg}$ twice daily in all cohorts. Vorinostat $100 \mathrm{mg}$ twice daily in cohort 1; $200 \mathrm{mg}$ in AM and $100 \mathrm{mg}$ in PM in cohort 2; $200 \mathrm{mg}$ twice daily in cohort 3 . Each cycle consisted of 14 days of treatment and 7 days of rest.

Table 2. Sor/vor/bor study patient characteristics

\begin{tabular}{|c|c|c|c|c|c|c|}
\hline $\begin{array}{c}\text { Phase } \\
1\end{array}$ & Patient & Age/Sex & AML status & Cytogenetics & FLT3-ITD & Response \\
\hline \multirow[t]{4}{*}{ Cohort 1} & 1 & $33 / \mathrm{M}$ & Rel post transplant & $(-7)$ & 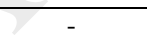 & Unevaluable \\
\hline & 2 & $67 / \mathrm{M}$ & $\begin{array}{l}\text { Rel/Ref post } \\
\text { transplant }\end{array}$ & $(-7 q)$ & + & Unevaluable \\
\hline & 3 & $64 / \mathrm{F}$ & Ref & Complex & - & No \\
\hline & 4 & $54 / \mathrm{F}$ & $\mathrm{Rel} / \mathrm{ref}$ & Complex & - & No \\
\hline \multirow{3}{*}{ Cohort 2} & 5 & $55 / \mathrm{F}$ & Rel/ref & Normal & + & CRi \\
\hline & 6 & $67 / \mathrm{M}$ & $\mathrm{Rel} / \mathrm{ref}$ & Normal & + & CRi \\
\hline & 7 & $64 / \mathrm{M}$ & Ref & Normal & + & Initial response \\
\hline \multirow{4}{*}{ Cohort 3} & 8 & $35 / \mathrm{M}$ & Ref & Unavailable & + & Unevaluable \\
\hline & 9 & $73 / \mathrm{M}$ & Ref & Complex & - & No \\
\hline & 10 & $51 / \mathrm{M}$ & Rel & Complex & - & No \\
\hline & 11 & $63 / \mathrm{M}$ & Ref & Complex & - & No \\
\hline \multirow{3}{*}{ Cohort 4} & 12 & $46 / \mathrm{F}$ & Ref & $46, \mathrm{XX}, \mathrm{t}(2 ; 8)$ & + & Initial response \\
\hline & 13 & $50 / \mathrm{F}$ & Ref & Normal & + & $\mathrm{CRi}$ \\
\hline & 14 & $37 / \mathrm{M}$ & Rel post transplant & Unavailable & + & $\mathrm{CRi}$ \\
\hline \multirow{3}{*}{ Cohort 5} & 15 & $40 / \mathrm{F}$ & Ref & Unavailable & + & No \\
\hline & 16 & $46 / \mathrm{M}$ & Ref & (-7)/Complex & - & No \\
\hline & 17 & $24 / \mathrm{F}$ & Rel post transplant & $46, \mathrm{XX}, \mathrm{t}(12 ; 15)$ & + & $\mathrm{CRi}$ \\
\hline \multirow{3}{*}{$\begin{array}{c}\text { Phase } \\
2\end{array}$} & $\begin{array}{c}7 \\
\text { patients }\end{array}$ & $\begin{array}{c}2 \text { Females } \\
5 \text { Males } \\
\text { Age } 41-72 \\
\end{array}$ & $\begin{array}{c}4 \mathrm{Rel} / \mathrm{ref} \\
1 \mathrm{Ref} \\
2 \mathrm{Rel} \text { post transplant }\end{array}$ & Normal & + & $\begin{array}{l}1 \mathrm{CR} \\
1 \mathrm{CRi} \\
2 \mathrm{PR}\end{array}$ \\
\hline & $\begin{array}{c}7 \\
\text { patients }\end{array}$ & $\begin{array}{l}3 \text { Females } \\
4 \text { Males } \\
\text { Age } 50-71\end{array}$ & $\begin{array}{c}5 \mathrm{Rel} / \mathrm{ref} \\
2 \mathrm{Rel} \text { post transplant }\end{array}$ & $\begin{array}{l}\text { Complex } \\
\text { (-5) or (-7) }\end{array}$ & - & No \\
\hline & $\begin{array}{c}6 \\
\text { patients }\end{array}$ & $\begin{array}{l}\text { 4 Females } \\
2 \text { Males } \\
\text { Age 54-73 }\end{array}$ & $\begin{array}{l}3 \mathrm{Rel} / \mathrm{ref} \\
3 \mathrm{Ref}\end{array}$ & $\begin{array}{l}2 \text { Normal } \\
3 \text { Complex } \\
1(-5)\end{array}$ & $\begin{array}{l}2+ \\
4-\end{array}$ & $\begin{array}{l}\text { Unevaluable } \\
3 \text { discontinued due to patient preference } \\
2 \text { died due to neutropenic fever } \\
1 \text { lost to followup }\end{array}$ \\
\hline
\end{tabular}

Rel: Relapsed; Ref: Refractory; CRi: Complete remission with incomplete platelet recovery

Dosing: Sorafenib $200 \mathrm{mg}$ twice daily in cohort 1 and $400 \mathrm{mg}$ twice daily in next cohorts. Vorinostat $200 \mathrm{mg}$ twice daily on days 1-4 and 8-12

in cohorts $1-4$, and continuously in cohort 5 . Bortezomib $1.0 \mathrm{mg} / \mathrm{m} 2$ on days 1,8 in cohorts 1 and $2 ; 1.3 \mathrm{mg} / \mathrm{m} 2$ on days 1,8 in cohort 3 ; and 1.3 $\mathrm{mg} / \mathrm{m} 2$ on days $1,4,8,11$ in cohorts 4 and 5 . Each cycle consisted of 14 days of treatment and 7 days of rest. 